\title{
Type I collagen degradation does not diminish with RA disease duration
}

\author{
M Hakala, K Aho, S Åman, R Luukkainen, M Kauppi, J Risteli
}

\begin{abstract}
Objective-To assess the relation between type I collagen degradation and the duration of rheumatoid arthritis (RA).

Methods-The serum concentrations of cross linked carboxyterminal telopeptide of type I collagen (ICTP) measured earlier in a community based series (90 patients) and a hospital based series (59 patients) were re-evaluated with reference to the duration of RA.

Results-The serum ICTP showed a positive correlation with the duration of the disease in the hospital based series $(r=0.40, p<0.01)$ but not in the community based one $(r=0.18, p=0.10)$.

Conclusions-Type I collagen degradation
Cons Conclusions-Type I collagen degradation
predominantly reflecting pathological bone destruction does not seem to diminish in longlasting RA.

(Ann Rheum Dis 2001;60:420-422)
\end{abstract}

Plain radiographs are considered the gold standard for measuring joint destruction in rheumatoid arthritis (RA). Erosions in periphrheumatoid arthritis (RA). Erosions in periph-
eral joints are one of the most characteristic
features of RA. Changes are easy to see, and features of RA. Changes are easy to see, and they provide a permanent record. Progression of the disease can be measurth anteroposterior serial radiographs, usually

The progression of radiographic changes in a number of inception cohorts has been followed up for up to five to eight years, but only limited information is available of longer follow up. Rasker and Cosh assessed the radiographic damage of metacarpophalangeal and wrist joints after 15 years. $^{2}$ Another long term study joints after 15 years. ${ }^{2}$ Another long term study
dealing with radiographic progression was cardealing with radiographic progression was car-
ried out by Scott et al. ${ }^{3}$ More recently, three further studies have been published. ${ }^{4-6}$ The criteria used in these studies were seldom fully comparable with each other.

Overall, the studies suggest that the most rapid advance in radiographic damage occurs during early disease. ${ }^{1}$ The curve of radiological damage ranges from a linear one in the early stages of the disease to a more level rate of progression at the later stages. Yet there is great variation in the type of progression between individual patients. ${ }^{5} \mathrm{~A}$ key question is whether the apparent changes in RA progression are actually artefactual and related to the ceiling effects inherent in radiographic readings. ${ }^{6}$ In addition, one limitation of radiographic studies is that they focus on the same joint areas, such as hands and feet, over time, though the disease process usually afflicts several joint areas with variable severity.
In recent years there has been an effort to develop biochemical tests to assess tissue destruction, such as degradation of cartilage and bone. One such test is radioimmunoassay for the carboxyterminal telopeptide of type I collagen $(\mathrm{ICTP})^{7}$ applicable to serum and synovial fluid. As recently reviewed, a number of prospective and cross sectional studies of both early and advanced RA have recorded with RA and shown concentrations in patien with RA and shown significant correlation between the ICTP concentration and radiographic joint damage. ${ }^{8}$ We have now reexamined the data of two earlier study series ${ }^{9} 1$ to answer the question: Does the degradation of type I collagen vary during the course of RA?

\section{Patients and methods}

Of the series of patients with RA re-evaluated for this study, one was community based and the other hospital based..$^{90}$ The former series consisted of 90 consecutive patients with RA ( 31 men, 59 women), who were the subjects of study on the medicosocial aspects of rheumatic diseases carried out in 1989-91 in the Kuusamo area, northern Finland, with 18000 inhabitants. $^{9}$ The patient population included approximately $85 \%$ of all subjects with RA in the area. The mean age of the patients was 58.7 years and the mean duration pers. Forty five $(76 \%)$ of the women were postmenopausal.

The hospital based series comprised 59 patients with RA (20 men, 39 women), who had been treated in three rheumatology units in Finland. ${ }^{10}$ The mean age of the patients was 58.1 years and the mean duration of the disease 13.5 (range $0.5-58$ ) years. Thirty one $(79 \%)$ of the women were postmenopausal.

ICTP was measured by equilibrium radiommunoassay ${ }^{7}$ with reagents supplied by Orion Diagnostica (FIN-90460 Oulunsalo, Finland).

The data were recorded and calculated on personal computer using the SOLO statistical software. Mann-Whitney test, $\lambda^{2}$ statistics, and Spearman's rank correlation coefficient test were used as appropriate.

\section{Results}

The serum ICTP concentration correlated positively with the duration of the disease in the hospital based series $\left(r_{\mathrm{s}}=0.40, \mathrm{p}<0.01\right)$, but not in the community based series $\left(r_{\mathrm{s}}=0.18\right.$, $\mathrm{p}=0.10$ ) (fig 1). There was also a correlation between age and ICTP in the hospital based series $\left(r_{\mathrm{s}}=0.47, \mathrm{p}<0.001\right)$, but not in the community based one $(r=0.08, \mathrm{p}=0.44)$. The and hospital based series were 4.5 (range 1.9- 

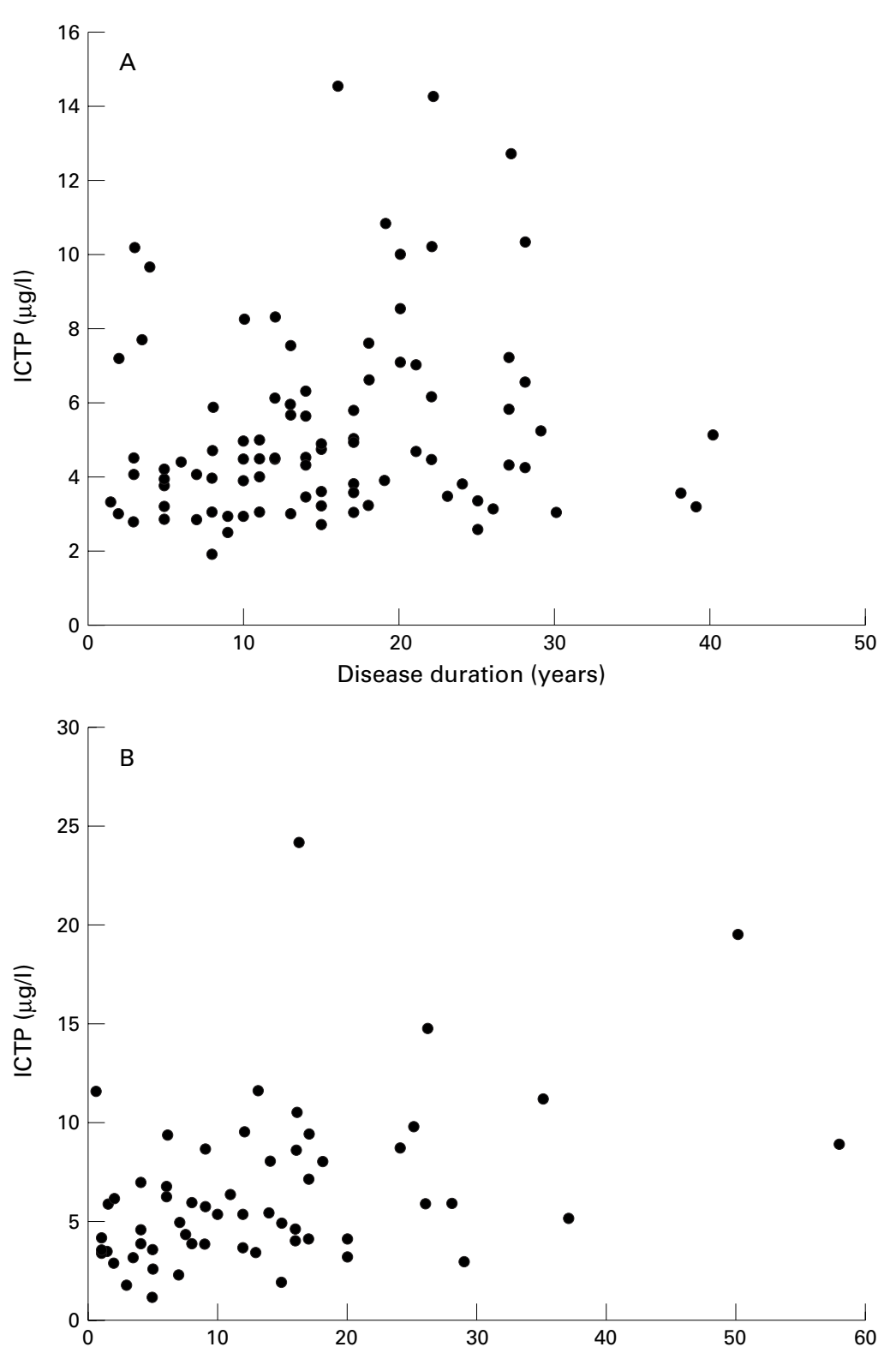

Disease duration (years)

Figure 1 Relation between serum carboxyterminal telopeptide of type I collagen (ICTP) and disease duration in two series of patients with rheumatoid arthritis: $(A)$ a co
based one $(r=0.18, p=0.10)$ and $(B)$ a hospital based one $(r=0.40, p<0.01)$.

$14.5)$ and $5.3(1.1-24.2) \mu \mathrm{g} / \mathrm{l}$, respectively. Neither series showed any significant differences in serum ICTP between men and women. There was also a closer correlation between ICTP and markers of disease activity, such as $C$ reactive protein and the joint swelling score, in the hospital based series $\left(r_{\mathrm{s}}=0.59\right.$, $\mathrm{p}<0.001 ; r_{\mathrm{s}}=0.65, \mathrm{p}<0.001$, respectively) than in the community based one $\left(r_{\mathrm{s}}=0.40\right.$ $\mathrm{p}<0.001 ; r_{\mathrm{s}}=0.38, \mathrm{p}<0.001$, respectively).

Discussion

Type I collagen accounts for about $90 \%$ of the organic matrix of bone. Tests reflecting its degradation have proved to be useful in assessing bone metabolism. ${ }^{11}$ Several immunoassays have been developed for structures involving the ICTP, and they give different results, depending on their immunochemical specificity for the size of the antigen of the cross links. ${ }^{11}$ Also the enzymes digesting type I collagen are important. Normal osteoclastic bone collagen degradation is mediate by cathepsin K, which destroys the ICTP antigenicity. ${ }^{11}$ Thus increased concentrations of ICTP reflect other routes of che likely those egradation, most tikely those mediated hrough matrix metalloproteinases.

The ICTP assay has turned out to be a reliable marker for increased type I collagen degradation in situations that include local destruction of bone tissue, such as multiple myeloma ${ }^{12}$ bone metastases from carcino${ }^{13}$ and both early and advanced RA ${ }^{8}$ On and the circulating ICTP antigen evels do not reflect accelerated or retarded physiological bone resorption, such as is seen in the postmenopausal state or during the use of oestrogen replacement therapy. ${ }^{14}$ No good methods are available for measuring the breakdown products of type II (cartilage) collagen.

In the work described here we reanalysed the Ierum ICTP data from two cross section series of patients with RA, one of them community based ${ }^{9}$ and the other hospital based..$^{10}$ Serum ICTP showed a positive correlation with the duration of the disease in the hospital based series but not in the community based one. Over time some patients with RA go on to have remission and are unlikely to be on to have remission and are unlikely to be parients with persistenty active, severe patients with persistently active, severe join disease- that is, with disease characteristics known to be reflected in the serum ICTP level ${ }^{9}$ - accumulate among hospital patients. On the other hand, a selective loss of severe cases probably occurs with time in the community based series owing to excess com com his study a more pronounced correlation was found between serum ICTP and markers of disease activity in the hospital based series than in the community based one.

The ICTP concentrations are higher in children than in adults. The manufacturer of the test kit recommends the same reference values for the whole adult age range, though marginal increase in serum ICTP takes place in marginal increase in serum ICTP takes place in
women after the menopause. ${ }^{14}$ We used ICTP values without any correction. Perhaps the diference between a patient's ICTP concentration and that of controls matched for age and ex might provide a more accurate estimate of ( arge control series with an age distribution similar to that of our patients with RA was available.

As it now stands, our data are in accordance with the view that pathological bone resorption does not diminish in longlasting RA. Wolfe and Sharp similarly reached the conclusion that pic in $\mathrm{RA}$ at a constant rate. ${ }^{6}$

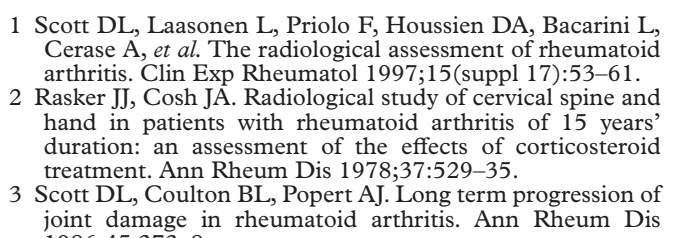

joint damage in rheumatoid arthritis. Ann Rheum Dis
1986;5:373-8. 
4 Kaarela K, Kautiainen H. Continuous progression of radioRheumatol 1997:24:1285-7.

5 Graudal N, Jurik AG, de Carvalho A, Graudal $H$. Radiographic progression in rheumatoid arthritis. A
long-term prospective study of 109 patients. Arthritis long-term prospective study of 109 patients. Arthritis
Rheum 1998;41:1470-80. 6 Wolfe F, Sharp JT. Radiographic outcome of recent-onset
arthritis. A 19-year study of radiographic progression. arthritis. A 19-year study of radiographic progression.
Arthritis Rheum 1998;41:1571-82. 7 Risteli J, Elomaa I, Niemi S, Novamo A, Risteli L. Radioimmunoassay for the pyridinoline cross-linked carboxyterm-
nal telopeptide of type I collagen: a new serum marker o Aman S. Markers of collagen metabolism in the assessmen of rheumatoid arthritis- - with special reference to cross-
linked carboxyterminal telopeptide of type I collagen linked carboxyterminal telopeptide of type I collagen
(ICTP) [thesis]. Acta Universitatis Ouluensis D Medica 532. Oulu: University of Oulu, 1999 . 9 Hakala M, Risteli L, Manelius J, Nieminen P, Risteli Increased type I collagen degradation correlates with
disease severity in rheumatoid arthritis. Ann Rheum Dis 1993;52:866-9.
0 Hakala M, Åman S, Luukkainen R, Risteli L, Kauppi $M$ metabolism in serum and synovial fluid for assessment of disease process in patients with rheumatoid arthritis. An Rheum Dis 1995;54:886-90.

Sassi M-L, Eriksen H, Risteli L, Niemi S, Mansell J, Gowen $\mathrm{M}$, et al. Immunochemical characterization of assay for the of antigenicity by treatment with cathepsin K. Bone 2000; 26:367-73.

Elomaa I, Virkkunen P, Risteli L, Risteli J. Serum concentration of the cross-linked carboxy terminal telopep-
tide of type I collagen (ICTP) is a useful prognostic indica-

3 Aruga A, Kolzumi M, Hotta R, Takahashi S, Ogata E. Use follow- of of bone metastasis from lung cancer. Br J Cancer 1997;76:760-4. Hassager C, Risteli J, Risteli L, Christiansen C. Effect of the
menopause and hormone replacement therapy on the menopause and hormone replacement therapy on the
carboxy-terminal pyridinoline cross-linked telopeptide of
type I collagen. Osteoporos Int 1994;4:349-52. 